\title{
GKL Tagung zur Bestandesaufnahme von Mikro- und Makroplastik im Gartenbau
}

\section{Blanke ${ }^{1}$}

Eingegangen: 9. Januar 2020 / Angenommen: 21. August 2020 / Online publiziert: 27. Oktober 2020

(c) Der/die Autor(en) 2020

\section{Zusammenfassung}

Die GKL-Tagung zum Nachhaltigen Einsatz von Kunststoffen im Gartenbau im September 2019 bei FVG in Dernbach informierte über den aktuellen Wissensstand

1) Nach der GKL-Erhebung von Freyer und Lampe in 2018 wird der Einsatz von Agrarfolien (LD-PE einschl. Netze aus HD-PE) im Obstbau auf ca. 16.000ha und im Gemüsebau auf ca. 40.000ha (einschl. Vlies) geschätzt; die Daten sind stimmig mit den zeitgleichen Gartenbau-Anbaustatistiken des JKI in 2018 und BMEL in 2014 und entsprechen ca. $22 \%$ der Anbaufläche im Obstbau und ca. 29\% im Gemüsebau.

2) Bei 4,07 Mio.t Kompost jährlich mit einer Fracht von 817t Mikroplastik liegen die Mikroplastik Einträge für die ca. 200.000ha Obst- und Gemüsebaufläche (einschl. Baumschulen) in Deutschland im theoretischen Durchschnitt bei ca. $61 \mathrm{t} / \mathrm{Jahr}$.

3) Diese Kunststoffeinträge mit dem Kompost lassen sich durch eine Änderung des Verhaltens der Verbraucher reduzieren, die ihren Biomüll in Plastiktüten sammeln und dann mit diesen und ebenso Blumen mit (Plastik-) Blumentopf in der braunen Biotonne entsorgen - dazu bedarf es Aufklärung durch den Grünen Punkt, die in Planung ist;

4) Für gebrauchte Hagelnetze (HD-PE) ist eine Ausdehnung des bereits am Bodensee bestehenden Rückführungssystems auf die anderen Obstbauregionen geplant sowie eine Ausweitung der Rückführung (und Recycling) von Agrarfolien im bestehenden (freiwilligen) ERDE Rückführsystem europäischer Kunststoffhersteller, so dass die Entstehung von Mikroplastik aus Agrarfolien bei der Folien-Entsorgung vermieden wird.

Insgesamt kann eine validierte Einschätzung der Emissionen von Mikroplastik aus Agrarfolien noch nicht vorgenommen werden und erfordert weitere Forschung sowie eine Recherche von Daten, die schwierig zu erheben sind, und vor allem Kenntnisse über die Abbauraten von Mikro- und Makroplastik in unterschiedlicher Umgebung.

Schlüsselwörter Entsorgung · Folienabdeckung · Hagelnetz · Kunststoff · Mulchfolien · Nachhaltigkeit · Polyolefine · Recycling $\cdot$ Ressourcenschutz $\cdot$ Zirkuläre Landwirtschaft

M. Blanke

mmblanke@uni-bonn.de

1 INRES-Gartenbauwissenschaft, Universität Bonn, Auf dem Hügel 6, 53121 Bonn, Deutschland 


\section{(Micro-)Plastics in Horticulture: GKL Workshop on Circular Economy of Plastics in Agriculture and Horticulture}

\section{Abstract}

The GKL September 2019 workshop on circular economy of plastics in agriculture presented data on the current use of mulch films, fleeces, foils and hail nets on ca. 16,000 ha of fruit cultivation and 40,000 ha of vegetables in Germany with the following breakdown

1) This is equivalent to $22 \%$ of the fruit and $29 \%$ of the vegetable acreage in Germany.

2) The annual compost usage of 4.07 mil tons in agriculture and horticulture in Germany includes $817 \mathrm{t}$ micro plastic.

3) Based on a $14 \%$ share, this is equivalent to ca. $61 \mathrm{t}$ microplastics per year for the 200,000 ha of fruit, nursery and vegetables grown in Germany.

4) The un-informed consumer carries a heavy burden, as the plastic in municipal compost largely originates from his disposed (black) plastic flower pots and carrier shopping bags used to collect organic household bio-waste.

5) A voluntary plastic collection system called 'ERDE' was set up in Germany by European Plastic manufacturers to collect any plastic waste from agriculture and horticulture for recycling into granules, thereby avoiding plastic waste in the countryside or sea.

Detailed values of plastic degradation in agriculture and horticulture into microplastics await ongoing studies.

Keywords Carbon sequestration · Circular economy · Compost · Crop cover · Hail net · Microplastics · Plastic mulch · Polyolefins $\cdot$ Recycling $\cdot$ Resource conservation $\cdot$ Sustainability

\section{Einleitung}

Die Tagung der Gesellschaft für Kunststoffe im Landbau (GKL) zum Nachhaltigen Einsatz und Ansätzen zur Kreislaufwirtschaft von Kunststoffen im Gartenbau und in der Landwirtschaft bei der Firma FVG in Dernbach/Westerwald Ende September wurde von Prof. Dr. Karl Schockert geleitet (Abb. 1).

Ziel war ein Überblick über erste Zwischenergebnisse, Eckdaten und Hochrechnungen aus laufenden Entwicklungen und Forschungsprojekten zu Agrarfolien (Mulchfolien, Tunnelfolien, Reflexionsfolien, Vliesen, Hagelnetzen) - und die Vorträge in der Expertenrunde kritisch in den

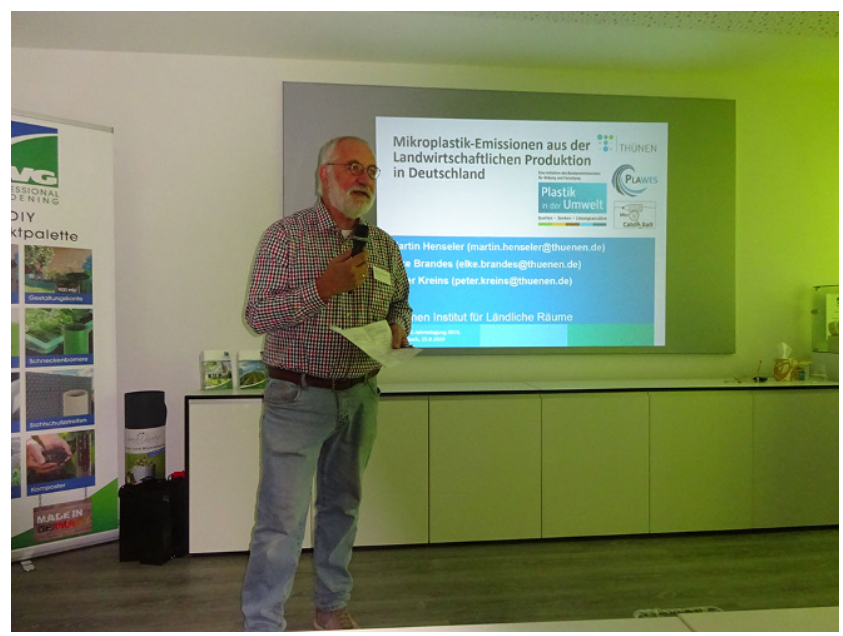

Abb. 1 Prof. K. Sckockert leitete die 48. GKL Tagung im September 2019
Kontext der Kreislaufwirtschaft der Kunststoffe (Abb. 2) einzuordnen.

\section{Weltweite und deutschlandweite Verbreitung von Kunststoff}

Auf der Erde gibt es zurzeit ca. 25 Mrd.t Kunststoff; pro Jahr kommen ca. 450 Mio.t Kunststoff dazu (Tab. 1), ein Teil davon wird nach seiner Nutzung zur Energiegewinnung

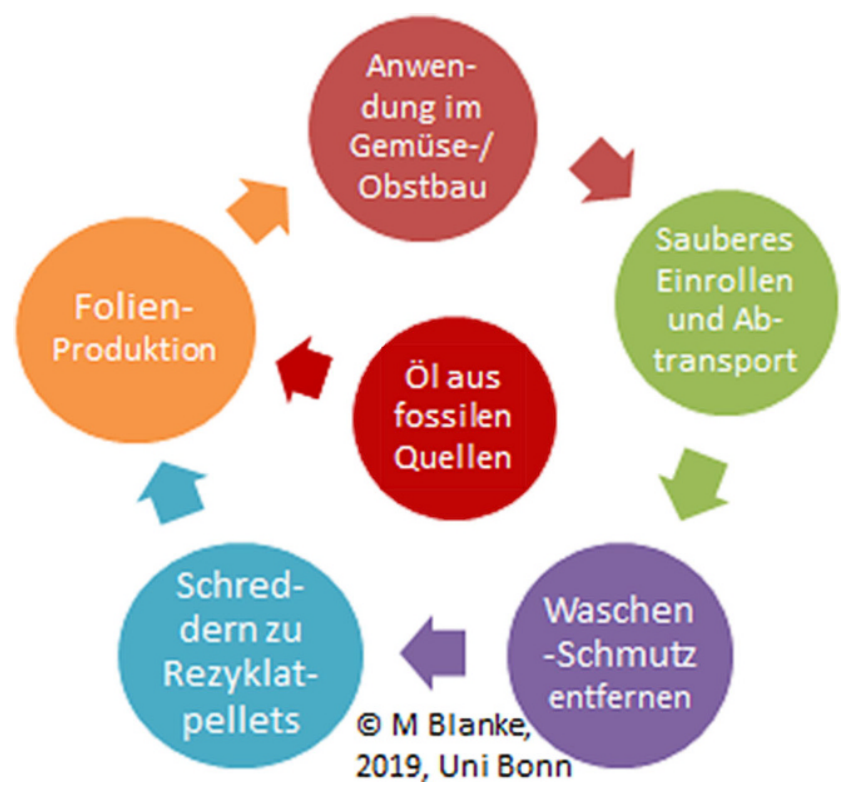

Abb. 2 Angestrebter Kreislauf der Kunststoffe in der Landwirtschaft und Gartenbau 
Tab. 1 Übersicht über die Kunststoffverbreitung weltweit und in Deutschland. (@ Plastics Europe 2018)

\begin{tabular}{ll}
\hline & Menge \\
\hline Weltweit & \\
Kunststoffverbreitung auf dem Planeten & $25 \mathrm{Mrd} . \mathrm{t}$ \\
Kunststoffherstellung pro Jahr & $450 \mathrm{Mio.} \mathrm{t} / \mathrm{Jahr}$ \\
Deutschland & \\
Kunststoffproduktion in Deutschland & $70.000 \mathrm{t} / \mathrm{Jahr}$ \\
Recycelt in 2018 & $17.000 \mathrm{t} / \mathrm{Jahr}$ \\
\hline
\end{tabular}

thermisch entsorgt und dient der Strom- oder Wärmegewinnung. In Deutschland werden pro Jahr ca. 70.000 t Kunststoffe hergestellt und ca. 17.000t recycelt, in Deutschland wird etwa die Hälfte des Kunststoffabfalls wiederverwertet.

\section{Definition und Ursprung von Mikroplastik: Autoreifenabrieb als Hauptverursacher}

Aus dem aktuellen Forschungsvorhaben bzw. der Konsortialstudie Kunststoffe von Juni 2018 des Fraunhofer-Institutes UMSICHT in Oberhausen stellten Mona Duhme und Ralf Bertling Hochrechnungen zu den möglichen Mikroplastik-Emissionswerten vor (Tab. 2). Unter Mikroplastik versteht man Partikel und Fasern aus Kunststoff ohne Größenbeschränkung; Mikroplastik akkumuliert. Die primäre Mikroplastik vom Typ A ist in Gebrauchsmitteln, die vom Typ B entstehen entweder erst bei Gebrauch oder erst mit der Zeit. Nach heutigem Wissensstand macht dabei der Abrieb der Autoreifen (Typ B) mit ca. 100.000t Mikroplastik/ Jahr den mit $87 \%$ überwiegenden Anteil von Mikroplastik aus, gefolgt von Kosmetika/Körperpflegemitteln (Mikroplastik Typ A); hinzu kommt Mikroplastik aus Abrieb durch Schuhsohlen, Waschen von Textilvlies u.a.. Nach dieser Konsortialstudie emittiert jeder Deutsche pro Jahr ca. 5,4 kg Plastik, davon 1,4 kg Makroplastik (26\%) und ca. $4 \mathrm{~kg}$ Mikroplastik (74\% bzw. 330.000 t/Jahr).

Diese Werte in Tab. 2 sind höher als die von 5 ebenfalls in dieser Konsortialstudie von Juni 2018 zitierten Autoren mit Werten um 1,5-3,0 kg/Kopf/Jahr. Wie umstritten diese Berechnungen sind, zeigte auch die Diskussion um Mikroplastik auf Sportplätzen mit Kunstrasen nach einer UMSICHT-Studie. Die genauen Ergebnisse des laufenden imulch-Projektes zum Verhalten von Mulchfolien Polyme-
Tab. 2 Typen und Emissionsquellen von Mikroplastik (@) Fraunhofer UMSICHT 2018) - Schätzungen und Hochrechnungen aus der Anzahl der Kfz und ihrer Jahresfahrleistung

\begin{tabular}{lll}
\hline Mikroplastik-Typen & Material & Emissionen \\
\hline Primäres Mikroplastik Typ A & $\begin{array}{l}\text { Kosmetika, Kör- } \\
\text { perpflegemittel u. a. }\end{array}$ & $\begin{array}{l}0,5 \mathrm{~kg} / \\
\text { Kopf } / \mathrm{Jahr}\end{array}$ \\
$\begin{array}{l}\text { Typ B } \\
\text { Entstehung durch Gebrauch/ }\end{array}$ & Reifen (Abrieb) & $3,5 \mathrm{~kg} /$ \\
$\begin{array}{l}\text { Benutzung } \\
\text { Makroplastik }\end{array}$ & Verpackungen & $\begin{array}{l}\text { Kopf } / \mathrm{Jahr} \\
1,4 \mathrm{~kg} /\end{array}$ \\
& & Kopf $/ \mathrm{Jahr}$ \\
Summe Makro- und Mikro- & - & $5,4 \mathrm{~kg} /$ \\
plastik & & Kopf $/ \mathrm{Jahr}$ \\
\hline
\end{tabular}

ren in der Landwirtschaft werden zum Ende des Projektes 2022 vorgestellt.

\section{Mögliche Ursachen von Mikroplastikeinträgen/-emissionen im Gartenbau und in der Landwirtschaft}

Die UMSICHT Konsortialstudie von Juni 2018 wurde im Juli 2019 von der Bundesgütegemeinschaft Kompost (BGK 2019a) kritisiert, die die insgesamt ausgebrachten Kompostmengen von 5,9 Miot auf ca. 4,07 Mio.t/Jahr sowie die jährliche Fracht an Kunststoffen von 12.000 t bei UMSICHT um das 15 fache auf $817 \mathrm{t} / \mathrm{Jahr}$ korrigierte - und zwar für Kompost und Gärreste (BGK 2019a). Bei einem Anteil von $53 \%$ für Kompost in Gartenbau und Landwirtschaft bzw. 436t/Jahr ergibt sich bei ca. 14\% (1,8\% Erwerbsgartenbau, 2,7\% Sonderkulturen, 7,4\% Hobbygartenbau und 2,1\% Sonstige) (BGK 2019b) ca. 61t Mikroplastik/Jahr (Tab. 3) für die Flächen der ca. 200.000 ha Obst- (einschl. Baumschulen) und Gemüsebau in Deutschland, auf denen Kompost ausgebracht wird.

Der Anteil der mit Kompost in die Umwelt eingetragenen Mengen an Kunststoffen wurde von der BGK in 2019 auf ca. 0,1\% der Gesamt-Kunststoffeinträge geschätzt, so dass Kompost im Rahmen des Stoffkreislaufes ('Cicular economy') (Abb. 2) ein wertvolles Gut zur Nährstoffversorgung und Anreicherung der organischen Substanz bzw. Kohlenstoffs im Boden ('Carbon sequestration') bleibt (Blanke und Kirsch 2002). Klärschlamm wird im Gartenbau seit langem nicht mehr eingesetzt (Blanke und Kirsch

Tab. 3 Plastikfracht im Kompost für den Gartenbau - berechnet nach Angaben des BGK (2019a, 2019b)

\begin{tabular}{llll}
\hline Parameter & Wert & Anteil Kompostvermarktung im Gartenbau & Plastikfracht (14\%) \\
\hline Jährlich ausgebrachte & 4,07 Mio.t FM/ & $1,8 \%$ Erwerbsgartenbau & 61 t/Jahr \\
Kompostmenge & Jahr & $2,7 \%$ Sonderkulturen & \\
Gesamte Plastikfracht im & $436 \mathrm{t} / \mathrm{Jahr}$ & $7,4 \%$ Hobbygartenbau & \\
Kompost & & $2,1 \%$ Sonstige & \\
\hline
\end{tabular}


Tab. 4 Mögliche Ursachen von Mikroplastikeinträgen/-emissionen im Gartenbau und in der Landwirtschaft - Schätzung aufgrund von Hochrechnungen (keine Erhebung/keine Analysen). (๑ Thünen Institut)

\begin{tabular}{lllll}
\hline Mikroplastik & Hochrechnung & Relevanz & $\begin{array}{l}\text { MP Emissionen pro Anwendungsfläche } \\
(\mathrm{kg} / \mathrm{ha} / \mathrm{Jahr})\end{array}$ & $\begin{array}{l}\text { MP Emissionen pro LN (kg/ha/ } \\
\text { Jahr) }\end{array}$ \\
\hline Klärschlamm & 0,7 Mio.t auf 6\% LN & Gering & Bis 4 & 0,242 \\
Kompost & 5,9 Mio.t auf 5\% LN & Hoch & Bis 3 & 0,143 \\
(Mulch-) Folien & 30.000ha (0,2\% der LN) & Sehr hoch & Bis 7 & $0,04-0,017$ \\
\hline
\end{tabular}

$L N$ Landwirtschaftliche Nutzfläche, MP Mikroplastik

Die Maximalwerte beziehen sich auf die Hochrechnungen, z. B. einem Mikroplastikgehalt im Kompost von 0,04\% Gew.anteil i.d. TM und 6,7 t/ha jährliche Ausbringung in der Landwirtschaft

Tab. 5 Schätzung des Folieneinsatzes im deutschen Gartenbau aufgrund Befragungen der Offizialberatung durch Freyer und Lampe (2018). (Quelle (C) GKL Umfrage 2018/9)

\begin{tabular}{|c|c|c|c|c|}
\hline & Folientyp & Folienfläche & Anbaufläche $^{\mathrm{a}}$ & Prozent \\
\hline \multicolumn{5}{|l|}{ Gemüsebau } \\
\hline Gemüsebau & Mulchfolien & $15.000 \mathrm{ha}$ & $115.000 \mathrm{ha}$ & 13 \\
\hline Bleichspargel & Schwarz-weiße Doppelfolie & $25.700 \mathrm{ha}$ & 25.700 ha & 100 \\
\hline Summe Gemüsebau & & 40.700 ha & 141.000 & 29 \\
\hline \multicolumn{5}{|l|}{ Obstbau } \\
\hline \multirow[t]{2}{*}{ Erdbeeren } & Mulchfolie & $10.000 \mathrm{ha}$ & 19.400 ha gesamt, davon 1700 unter & - \\
\hline & Tunnelfolie & $1700 \mathrm{ha}$ & Folie & - \\
\hline Kern- und Steinobst & $\begin{array}{l}\text { Hagelnetz (Kernobst) und Tunnel (Stein- } \\
\text { obst) }\end{array}$ & 6000 ha & $\begin{array}{l}\text { Apfel } 34.000 \text { ha; Süßkirschen } \\
6000 \text { ha }\end{array}$ & - \\
\hline Summe Obstbau & & $16.000 \mathrm{ha}$ & 76.000 ha & 22 \\
\hline
\end{tabular}

${ }^{\mathrm{a} A n b a u f l a ̈ c h e n ~ n a c h ~ G a r m i n g, ~ D i r k s m e i e r ~ u n d ~ B o r k ~(J K I ~ A u g u s t ~ 2017) ~}$

2002) und Gärreste in sehr geringem Umfang (Blanke und Kirsch 2004).

Martin Henseler, Elke Brandes und Peter Kreins vom Thünen Institut stellten die beiden Forschungsprojekte Plawes und Catch_Balt mit ersten vorsichtigen Annahmen, Hochrechnungen bzw. Zwischenergebnissen vor, woher Mikroplastik im Gartenbau und Landwirtschaft kommen könnte (Tab. 4); die genauen Ergebnisse und Details werden nach Abschluss der beiden Projekte veröffentlicht.

Da bisher keine Werte für die Emissionen von Mikroplastik aus Agrarfolien vorliegen, wurden diese in Tab. 4 von den Autoren als 1 oder $5 \%$ (willkürlich) angenommen - dies führte zu einer heftigen Diskussion im Auditorium.

\section{Folieneinsatz (LDPE und HDPE) im Gartenbau zur Versorgung des Verbrauchers mit saisonalen, regionalen Produkten}

Isabelle Lampe stellte die Ergebnisse der letzten GKL Umfrage 2018/19 gemeinsam mit Frau Freyer zum Einsatz von Kunststoffen im deutschen Gartenbau vor - die Werte in Tab. 5 beruhen auf Schätzungen der regionalen Offizialberatung.

Setzt man diese Werte in Bezug zu den vom BMEL (2014) und von Garming et al. (2017) zeitgleich erhobenen Anbauflächen, so ist der Einsatz auf ca. $22 \%$ der Obstbau- flächen, nahezu $100 \%$ im Spargelanbau und auf ca. 13\% der Gemüsebau-Flächen zu finden, wobei bei Erdbeeren der Einsatz von schwarzer Mulchfolie im Tunnelanbau (Abb. 3) zu Doppelnennungen führte. Der geschützte Anbau von Erdbeeren im Tunnel (ca. 1700 ha nach Linnemannstöns 2018) und die Abdeckung mit schwarz-weißer Folie beim Spargel dient der Verfrühung dieser Kulturen, um die vom Konsumenten und Handel geforderten Produkte aus regionalem Anbau zur Verfügung zu stellen. Bei Süßkirschen (ca. 700 ha nach Balmer und Kockerols 2018) trägt der Folientunnel zur Vermeidung des Platzens der Früchte bei

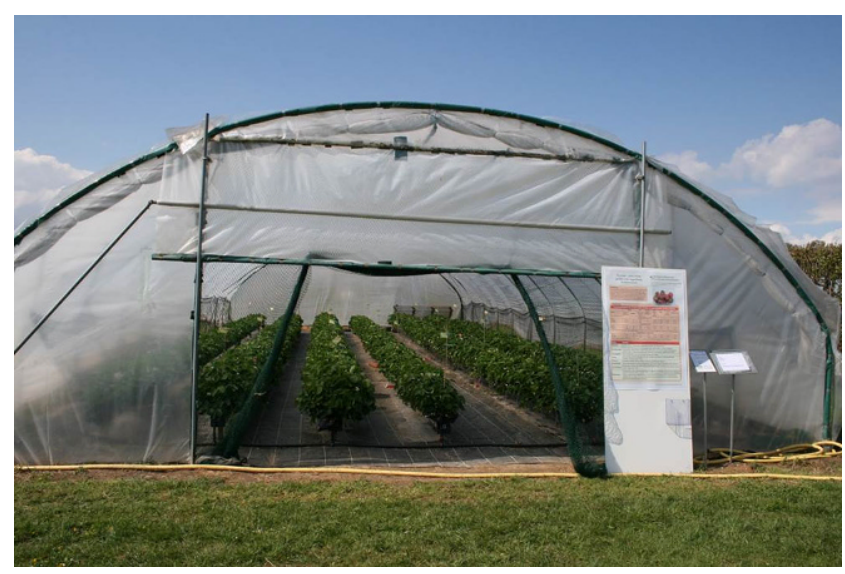

Abb. 3 Tunnelanbau für Erdbeeren. (@ C. Lankes, Uni Bonn) 


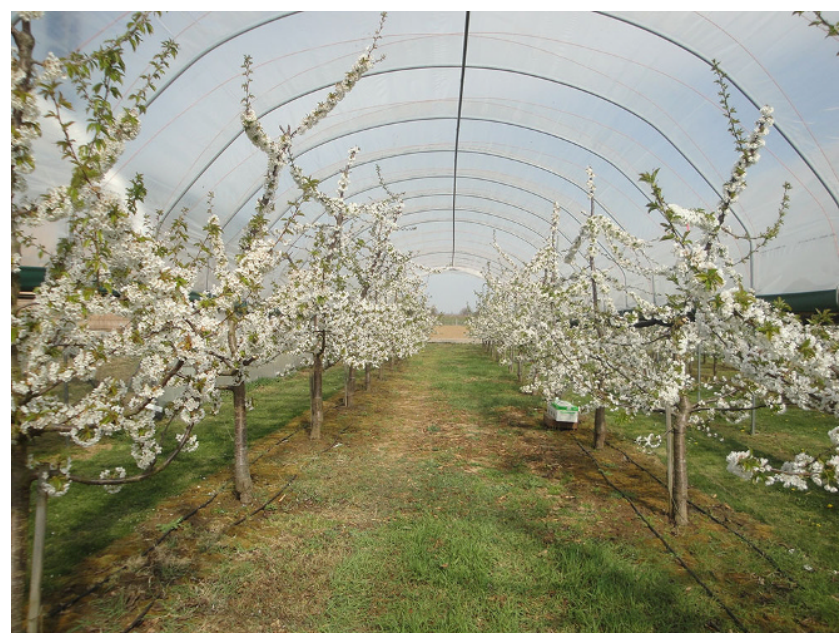

Abb. 4 Tunnelanbau für Süßkirschen. (@ M. Blanke, Bonn)

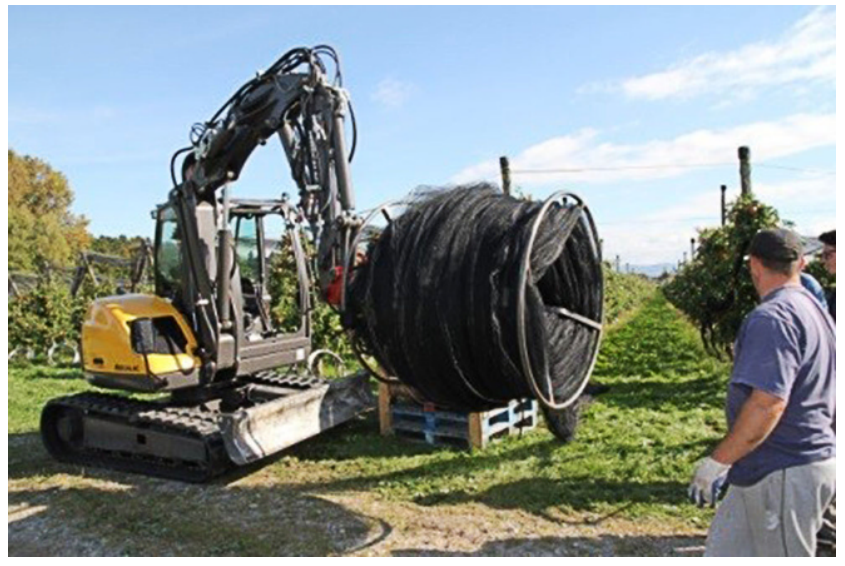

Abb. 5 Aufrollen gebrauchter Hagelnetze (HD-PE) zum Recyceln. (ㄷ R. Holzwarth, BayWa)

nasser Witterung bei (Abb. 4). Ohne diese Folien wären zu dieser Zeit eine Versorgung des deutschen Marktes bzw. Konsumenten nur mit Importware z. B. aus Spanien, Italien, Griechenland, Türkei oder Marokko möglich.

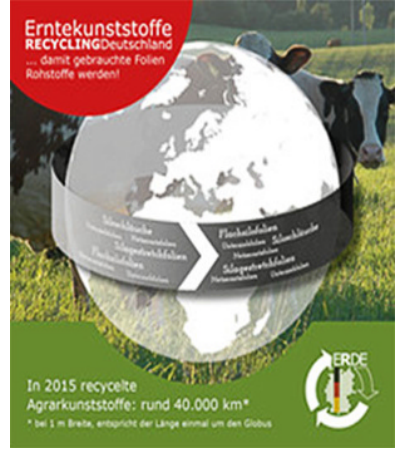

Abb. 6 Logo des ERDE Rückhol- und Recyclingsystem für Agrarfolien (LD-PE). (Beide (C) ERDE)

\section{Ausdehnung der Sammelsysteme für Hagelnetze}

Am Bodensee bestehen von der BayWa (Abb. 5) als auch der MaBo seit 2017 Rückholsysteme für verbrauchte Hagelnetze, die ab 2020 auf ganz Deutschland ausgedehnt werden sollen.

\section{'ERDE' zum Rückführen und Recycling von Folien aus Gartenbau und Landwirtschaft}

Von 70.000t geschätzter Jahresproduktion wurden 2019 ca. 17.000t Folien recycelt (Tab. 7). Die unterschiedliche Lebensdauer bzw. längere Lebensdauer der Folien als 1 Jahr (Tab. 6) erklärt einen Teil der Differenz zwischen beiden Werten.

Bei der erfolgreichen Rückführung von leeren und gesäuberten Kunststoffbehältern für Pflanzenschutzmittel ('PAMIRA'; 3500 t/Jahr, ca. 94\% Recyclingquote) ist die Teilnahme als Anreiz Bestandteil der QS/GlobalGAP Zertifizierung der Gartenbaubetriebe. In Anlehnung an das freiwillige 'PAMIRA' baut die RIGK ein freiwilliges Rückholsystem 'ERDE' (Erntekunststoffe Recycling DEutschland; www.erde-recycling.de) mit bisher 77 Sammelstellen auf; ERDE (Abb. 6) ist eine Initiative vieler europäischer Kunststoffhersteller.

Thomas Neck vom RIGK berichtete, dass 2018 13.000t Kunststofffolien (einschl. Vliese und Netze) aus Gartenbau und Landwirtschaft eingesammelt wurden, 2019 werden es ca. 17.000t sein (Abb. 7) mit Schätzung von 25.000t im Jahre 2022 (Tab. 7). Ab 2020 sollen auch schwarz-weiße

Tab. 6 Langlebigkeit: Geschätzte Haltbarkeit der Gartenbaufolien. (Blanke 2017)

\begin{tabular}{llll}
\hline Folientyp & Folienart & Einsatz & Haltbarkeit \\
\hline Mulchfolie & PE & Gemüse, Erdbeeren & 1-2 Jahre \\
Schwarze-weiße Mulchfolie & PE & Bleichspargel & 10 Jahre \\
Tunnelfolie & LD-PE & Süßkirschen (700 ha) & 1-2 Jahre \\
Hagelnetz & HD-PE & Kernobst & 8 (Kristallnetz) bis 20 Jahre (schwarz - mit Ruß - UV-verstärkt) \\
Reflexionsfolien & PP & Baumobst & 7-10 Jahre \\
\hline
\end{tabular}


Tab. 7 Rückführungsquoten von Kunststofffolien aus Gartenbau und Landwirtschaft. (C) Thomas Neck, RIGK, 2019)

\begin{tabular}{lll}
\hline Jahr & Folienrückführung & Quote \\
\hline 2018 & $13.000 \mathrm{t}$ & $16 \%$ \\
2019 & $17.000 \mathrm{t}$ & $20 \%$ \\
2020 & - & - \\
2021 & - & - \\
2022 & $25.000 \mathrm{t}$ & $65 \%($ Ziel $)$ \\
\hline
\end{tabular}

13.433

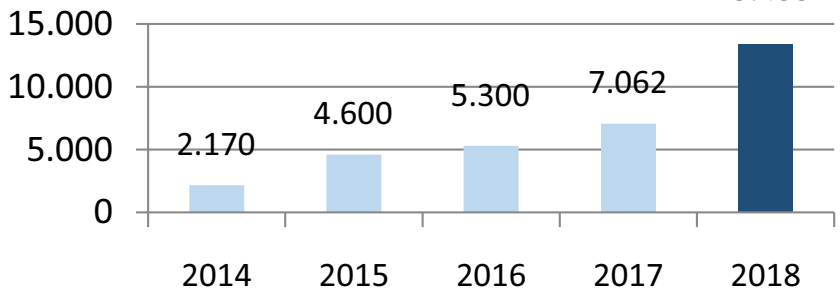

Abb. 7 Agrarfolien - Sammelmengen der ERDE - in Tonnen/Jahr

Taschenfolien aus dem Spargelanbau dazukommen, bei denen der hohe Verschmutzungsgrad mit Sand problematisch ist.

\section{Schwarze Kunststoff-Blumentöpfe schwierig auszusortieren}

Die Blumentöpfe, die die Verbraucher unachtsam mit der verwelkten Pflanze/Blume in der Biotonne entsorgen (Abb. 8), gehören mit zu den Ursprüngen von (Mikro-)Plastik im Kompost, in dem keine Kunststoffe ausgesondert werden.

Aber auch in der gelben Tonne bzw. im gelben Sack können schwarze Blumentöpfe und andere schwarze Plastikartikel wie leere Herren Shampoo-Behälter nicht aussortiert werden. Die schwarze Farbe absorbiert die NIR Strahlen im ersten Sortierdurchgang, die sonst eine Zuordnung zu PET, PP oder PE und Recycling ermöglicht.

Tab. 8 Alternativen zu schwarzen Blumentöpfen aus neuem Kunststoff

\begin{tabular}{ll}
\hline Ansatz & Beispiel \\
\hline Materialreduktion & Thermoplast, mtm \\
Farbige Blumentöpfe aus recyceltem & Blaue Serie der Fa. Pöppel- \\
PP/PE & mann \\
Pfand auf Blumentöpfe & $\begin{array}{l}\text { Gärtnerei Heino Schwarz, } \\
\text { Ansbach }\end{array}$ \\
Töpfe aus recyceltem Zellstoff & D-Grade Fibre Serie der Fa. \\
& Desch \\
Verottbare Töpfe aus Gras, Reis-, & D-Grade Serie der Fa. \\
Kokosschalen & Desch \\
\hline
\end{tabular}

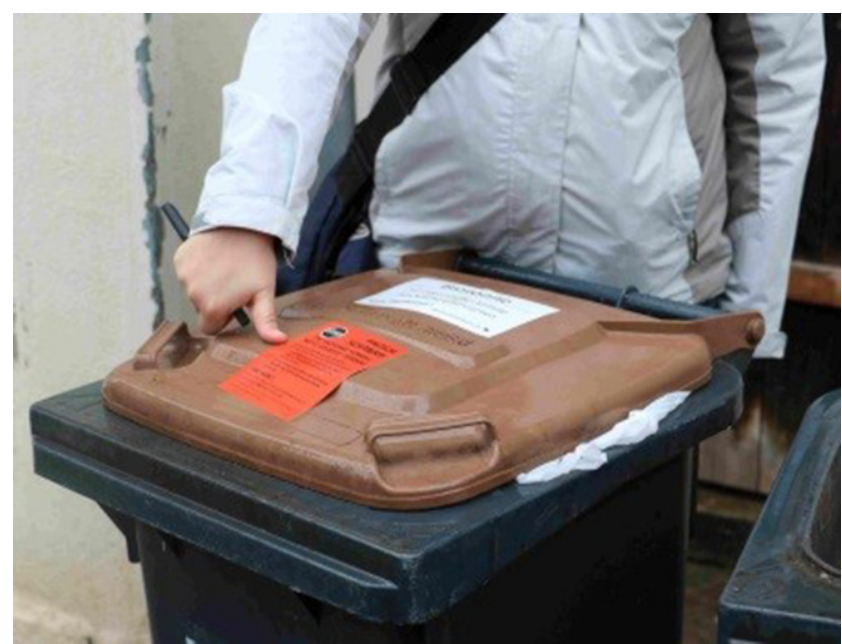

Abb. 8 Biotonnen, in die der Verbraucher Kunststoff entsorgt, werden nicht mehr überall geleert. (ㄷ H. Heinritz)

\section{Pfand für Blumentöpfe - eine individuelle innovative Lösung}

Die Gärtnerei Heino Schwarz im Landkreis Ansbach (Bayern) geht einen anderen Weg als die Verwendung recycelter Töpfe; sie erhebt 6 Cent Pfand für ihre eigenen mit Firmenlogo bedruckten Blumentöpfe (Abb. 14), ein Beitrag, der zur Finanzierung einer besonderen Topfmaschine notwendig ist. $\mathrm{Zu}$ Beginn dieser Aktion wurden ca. 30\% der Töpfe wieder verwendet - das Ziel ist $70 \%$; der Ausschuss unbrauchbarer Blumentöpfe beträgt ca. $5 \%$ (Homepage und pers. Mitteilung H. Schwarz, 2019). Durch die Wiederverwendung entfallen Sammlung, Abtransport und Wiederaufbereitung der alten Blumentöpfe mit vom Autor geschätzten 2 Mio.t $\mathrm{CO}_{2}$ eq Ersparnis pro Jahr.

\section{Sind Ersatz, Biokunststoff oder Recyclingprodukte die Lösung?}

Die längere Abbauphase bestimmter Biokunststoffe überschreitet die Kompostierungsphase vieler Kompostwerke von 2-3 Wochen, so dass die meisten Biokunststoffe nicht für die Biotonne geeignet sind. Biokunststoffe aus Mais stehen zudem in der Konkurrenz sowohl zur Nahrungsmittelproduktion als auch zu den Biogasanlagen.

Bei Rückführung der Gartenbaufolien durch ein System wie 'ERDE' werden die gebrauchten Folien an Zentralstellen eingesammelt, komprimiert, kompakt zu den Recycelbetrieben abtransportiert und dort durch Shreddern erst zu flakes (Abb. 9) und dann zu Recyclatpellets (Abb. 10) aufgearbeitet, so dass bei dieser Entsorgung kein Mikroplastik entsteht und dann $\mathrm{zu}$ den folgenden Produkten verarbeitet werden kann: 


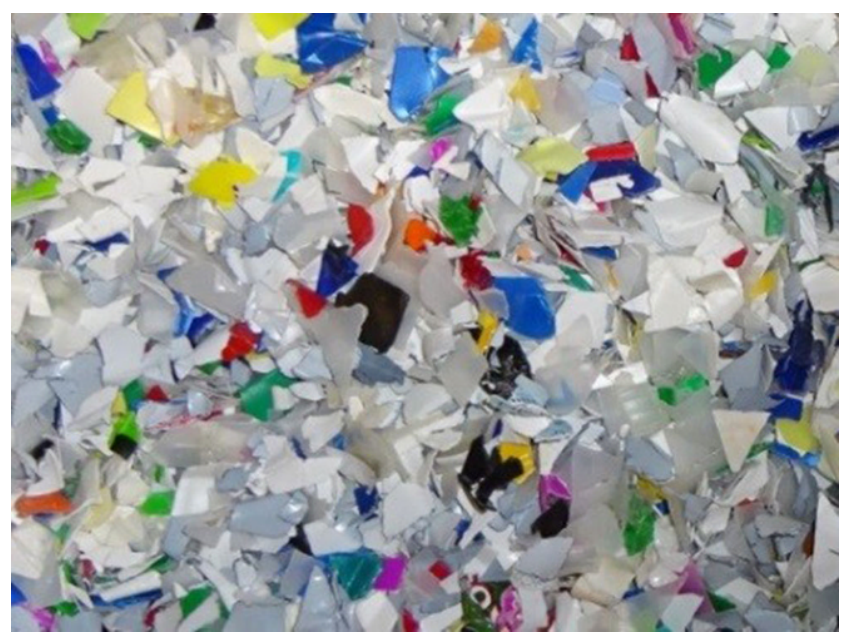

Abb. 9 Flakes als Vorstufe für Regranulat. (@ M. Blanke, Bonn)

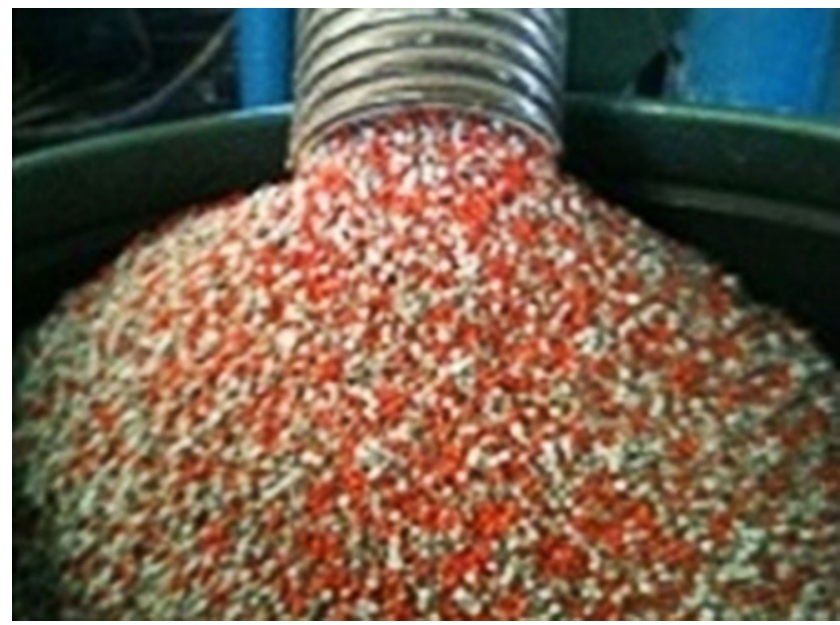

Abb. 10 Kunststoff-Pellets

\section{Produkte aus Recyklaten bzw. Polyolefinen (PP/PE)}

Die Palette von Produkten aus Recyclat reicht von Kompostern, Rasenkanten (Abb. 11 und 12), Schneckenbarrieren bis zu Wurzelsperren (Tab. 9), Topfhaltern von mtm bis zur „blauen Blumentopf-Serie“ der Fa. Pöppelmann (Abb. 13).

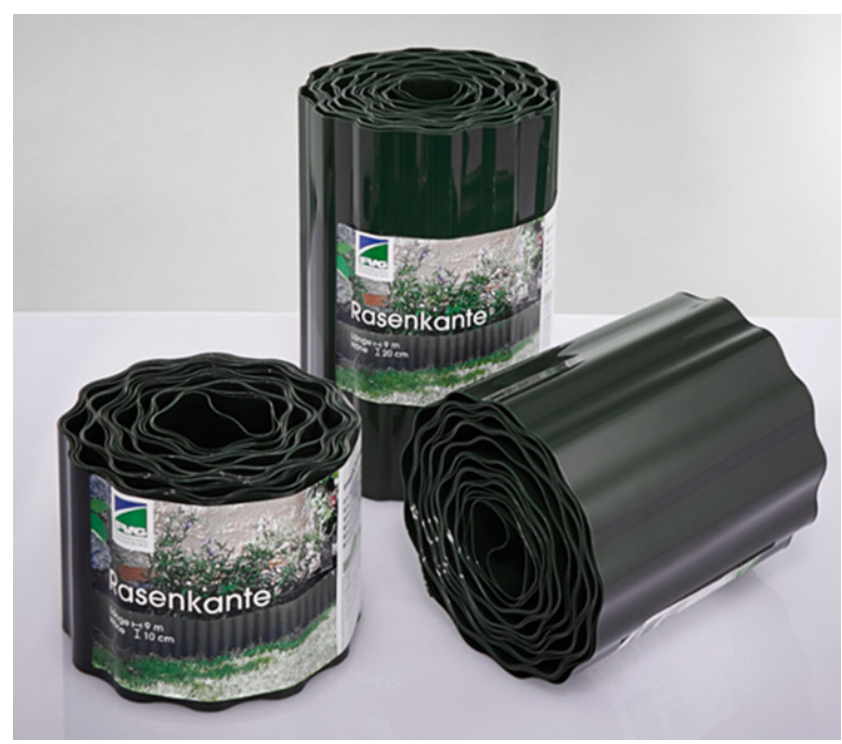

Abb. 11 Rasenkante der Fa. FVG. (Foto: H. Klar)

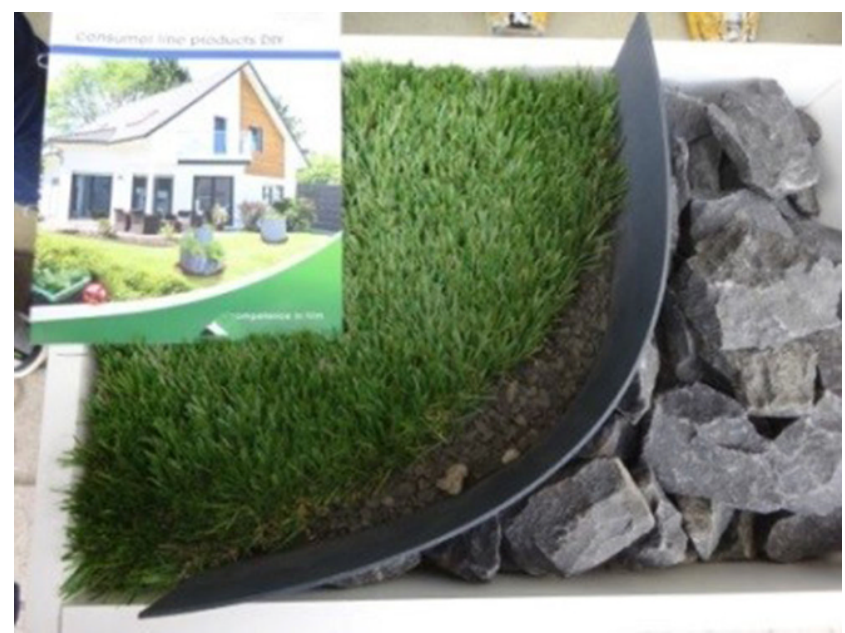

Abb. 12 Rasenkante der Fa. FVG. (Foto: M. Blanke)

\section{Strategien für den Kunststoffkreislauf - „Re-think plastics"}

Günther Orschulik (Fa. Pöppelmann) und Wouter Zieck (Fa. Desch) stellten in ihren Vorträgen ganzheitliche Kunststoffkreislauf-Konzepte (Tab. 8) wie „Re-think plastics“ vor:
Tab. 9 Beispiele für Produkte aus Polyolefinen (PP/PE) Recyclat

\begin{tabular}{llllll}
\hline Produkt & Recyclat & Anbieter & Produkt & Anbieter \\
\hline Rasenkante & PP/PET & FVG & Rasengitter & - & Mtm \\
Schneckenbarriere & PP/PET & FVG & Profile & - & Mtm \\
Wurzelsperre & PP/PET & FVG & Kisten, Tüllen & - & Mtm \\
Komposter & PP & FVG & Trays & - & Densch \\
Komposter & PP & mtm & Blumentopf & PE/PP & Pöppelmann \\
\hline
\end{tabular}




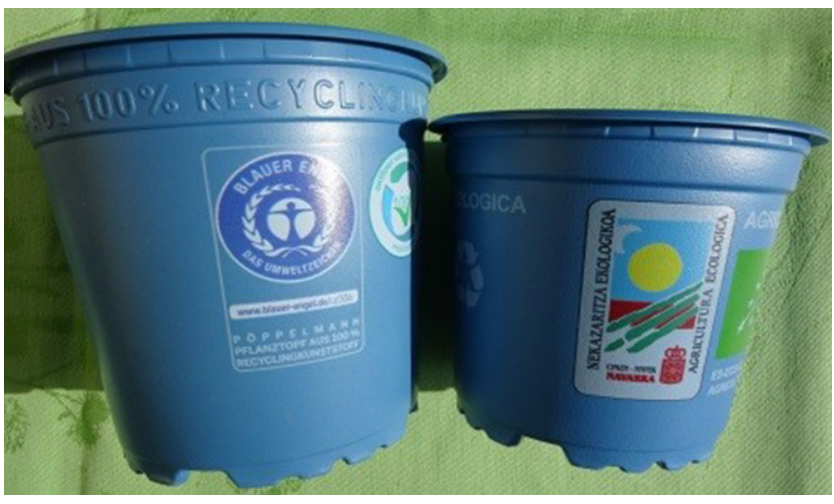

Abb. 13 Blauer Recycling-Blumentopf der Fa. Pöppelmann. (Photo: M. Blanke)

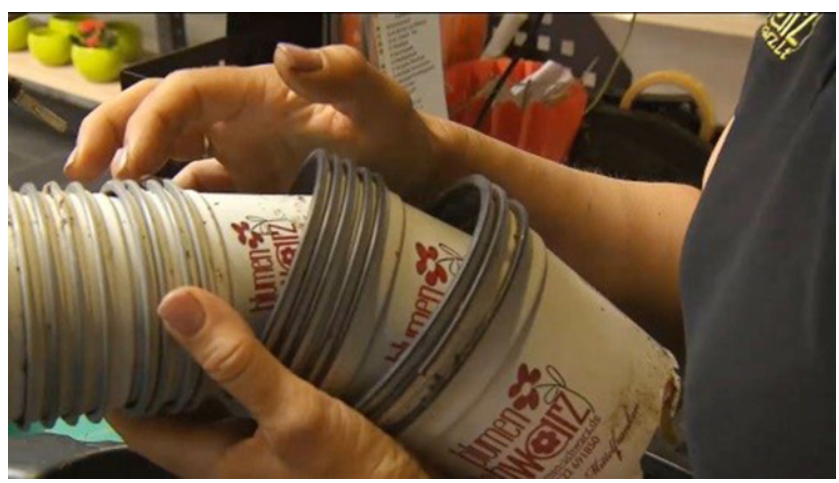

Abb. 14 Bedruckte Pfand-Blumentöpfe der Fa. Schwarz. (ㄷ BR 2019 und (C) H. Schwarz)

- Material und Energie (Grüner Strom) bei der Herstellung einsparen (z.B. Thermoformtöpfe)

- Langlebiger Kunststoff und Material wiederverwenden (Gärtnerei Schwarz, Abb. 14)

- bessere Recyclefähigkeit (Detektierbarkeit des Kunststofftyps, kein Schwarz, kein Kunststoffmix) (Fa Pöppelmann)

- mehr Kunststoff-Recycling (s. ERDE)

- alternative Materialien wie recycelter Papierzellstoff (z. B. Desch D-grade fibre) u.a.

\section{Markt für Recyklate}

Den Preis für Recyclate bestimmen Angebot, Nachfrage, Reinheit des Kunststoffes, Aufwand zur Reinigung und Verschmutzungsgrad. Bei R-PET (aus Recyclat) ist der Preis mit $€ 1100-1200 / t$ sogar teurer als neues PET, da sie gesondert mit geringem Schmutzanteil gesammelt werden und die Käufer den Mehrwert als Verkaufsanreiz und für Werbung ihrer umweltfreundlichen Produkte bzw. Verpackung nutzen - diese Situation ist bei verschmutzten PE-Mulchfolien im Moment nicht gegeben.

\section{Verbesserung des Carbon Footprint, Beitrag zum Klimawandel und Ersatz für Kunststoffexport nach China}

Thomas Neck von RIGK sprach von 9200t eingesparten $\mathrm{CO}_{2}$ durch die 2018 7000t eingesammelten Agrarfolien, „,weil durch die stoffliche Wiederverwertung einer Tonne des ERDE-Sammelgutes 1,3t $\mathrm{CO}_{2}$ weniger in die Atmosphäre als bei der Produktion einer entsprechenden Menge an Kunststoff-Neuware anfallen.:“ „Durch die eingeführten Importrestriktionen für Kunststoffabfälle in China kommt es zu einem Überangebot an Sekundärkunststoffen auf dem deutschen Markt. Dies führte zu einer erhöhten Auslastung der Recyclingkapazitäten und in Folge dessen zu erhöhten Entsorgungskosten für Kunststoffe. Obwohl Agrarkunststoffe aus dem ERDE-SYSTEM nicht nach China importiert wurden, sind auch in diesem Bereich deutliche Kostenerhöhungen eingetreten (RIGK) “.

Danksagung Ich danke Herrn Holzwarth (Fa. BayWa Tettnang) und den Firmen FVG, mtm u. Pöppelmann für die Fotos ihrer Recyclingprodukte aus Polyolefinen bzw. aus Kunststofffolien, Heino Schwarz für die Fotos der wieder verwendbaren Blumentöpfe, Dr. Christa Lankes für Abb. 4, Harald Heinritz der Abfallwirtschaft Kitzingen für Abb. 8, Dr. Andreas Kirsch (BGK Köln) für die Datenquelle zum Plastikgehalt in Kompost sowie Dr. Isabelle Lampe, Dr. Andreas Kirsch, Rudi Holzwarth und Henrik Klar für die kritische Durchsicht des Manuskriptes und wertvolle Anregungen und Dr. D.P. Hucklesby, Bristol, UK für die Korrektur des englischen Abstracts.

Funding Open Access funding enabled and organized by Projekt DEAL.

Interessenkonflikt M. Blanke gibt an, dass kein Interessenkonflikt besteht.

Open Access Dieser Artikel wird unter der Creative Commons Namensnennung 4.0 International Lizenz veröffentlicht, welche die Nutzung, Vervielfältigung, Bearbeitung, Verbreitung und Wiedergabe in jeglichem Medium und Format erlaubt, sofern Sie den/die ursprünglichen Autor(en) und die Quelle ordnungsgemäß nennen, einen Link zur Creative Commons Lizenz beifügen und angeben, ob Änderungen vorgenommen wurden.

Die in diesem Artikel enthaltenen Bilder und sonstiges Drittmaterial unterliegen ebenfalls der genannten Creative Commons Lizenz, sofern sich aus der Abbildungslegende nichts anderes ergibt. Sofern das betreffende Material nicht unter der genannten Creative Commons Lizenz steht und die betreffende Handlung nicht nach gesetzlichen Vorschriften erlaubt ist, ist für die oben aufgeführten Weiterverwendungen des Materials die Einwilligung des jeweiligen Rechteinhabers einzuholen.

Weitere Details zur Lizenz entnehmen Sie bitte der Lizenzinformation auf http://creativecommons.org/licenses/by/4.0/deed.de.

\section{Literatur}

Balmer M, Kockerols M (2018) Steinobstanbau unter Folie. GKL Jahrestagung Auweiler, 26. Sept. 2018 (Vortrag)

BGK (2019a) Kunststoffe in Kompost und Gärprodukten. Bundesgütegemeinschaft Kompost Köln (BGK), Köln 
BGK (2019b) Humus und Kompost aktuell. Q1, Abb. 4, S 6 online unter: https://www.kompost.de/fileadmin/user_upload/Dateien/ HUK-Dateien/2020/Q1_2020/Verwertung_von_Bioabfaellen_ 2019_HUK_Q1_2020.pdf

Blanke M (2017) Farbige Hagelnetze. Erwerbs-Obstbau 58:127-139. https://doi.org/10.1007/s10341-007-0048-6

Blanke M, Kirsch A (2002) Möglichkeiten und Grenzen des Komposteinsatzes im Obstbau. Kapitel 10. In: Kompost im Gartenbau, 1. Aufl. ZVG, Bonn, S 167-199

Blanke M, Kirsch A (2004) Möglichkeiten und Grenzen des Einsatzes organischer Sekundärrohstoffe im Obstbau. Müll-Handbuch. In: Hösel et al (Hrsg) Loseblatt-Sammlung, Lieferung 6550. ESVErich Schmidt Verlag, Berlin, S 1-12

BMEL (2014) Der Gartenbau in Deutschland - Daten und Fakten
Freyer G, Lampe I (2018) GKL Umfrage zum Einsatz von Folien im Gartenbau. Vortrag der Ergebnisse auf der GKL Jahrestagung Dernbach September 2019

Garming H, Dirksmeyer W, Bock L (2017) Entwicklungen des Obstbaus in Deutschland 2005 bis 2017. Thünen Working Paper Nr. 100

Linnemannstöns L (2018) Erdbeeren unter Folie. GKL Jahrestagung Auweiler, 26. Sept. 2018 (Vortrag)

Umsicht (2018) Kunststoffe in der Umwelt - Mikro und Makroplast. Konsortialstudie des Fraunhofer Institutes UMSICHT. Eigenverlag, Oberhausen 